This item was submitted to Loughborough's Research Repository by the author.

Items in Figshare are protected by copyright, with all rights reserved, unless otherwise indicated.

\title{
Self-assembly of hydrophilic homopolymers: a matter of RAFT end groups
}

PLEASE CITE THE PUBLISHED VERSION

http://dx.doi.org/10.1002/smll.201100382

\section{PUBLISHER}

(c) Wiley-VCH

\section{VERSION}

AM (Accepted Manuscript)

\section{PUBLISHER STATEMENT}

This work is made available according to the conditions of the Creative Commons Attribution-NonCommercialNoDerivatives 4.0 International (CC BY-NC-ND 4.0) licence. Full details of this licence are available at: https://creativecommons.org/licenses/by-nc-nd/4.0/

\section{LICENCE}

CC BY-NC-ND 4.0

\section{REPOSITORY RECORD}

Du, Jianzhong, Helen Willcock, Joseph P. Patterson, lan Portman, and Rachel K. O'Reilly. 2016. "Selfassembly of Hydrophilic Homopolymers: A Matter of RAFT End Groups". figshare.

https://hdl.handle.net/2134/22891. 


\section{suminustosmall}

DOI: $10.1002 /$ smll.((please add manuscript number))

Self-assembly of Hydrophilic Homopolymers: A Matter of End Groups?**

Jianzhong Du, ${ }^{a}$ Helen Willcock ${ }^{a}$ Joseph P. Patterson ${ }^{a}$ Ian Portman ${ }^{b}$ and Rachel K. O'Reilly

$a,{ }^{*}$

((Optional Dedication))

[*] Dr. R.K. O’Reilly, Dr. J. Du, Mr J.P. Patterson, Dr. H. Willcock

Department of Chemistry

Gibbet Hill Road,

University of Warwick

CV4 7AL (UK)

E-mail: r.k.o-reilly@warwick.ac.uk

Dr. N. Wilson

Department of Life Sciences

Gibbet Hill Road,

University of Warwick

CV4 7AL (UK)

Supporting Information is available on the WWW under http://www.small-journal.com or from the author.

Keywords: homopolymers, self assembly, vesicles, RAFT, end groups

\section{Abstract:}

Unusual self-assembly behavior is observed for a range of hydrophilic homopolymers.

This self-assembly behavior is contrary to the expected behavior of such hydrophilic polymers and instead mimics more commonly reported amphiphilic block

copolymers. It is proposed that the unique combination of hydrophobic end groups

at both the $\alpha$ and $\omega$ chain end accounts for this unusual self-assembly behavior.

Complex internal polymer micelles are spontaneously formed when hydrophilic

homopolymer polyelectrolytes and neutral polymers (with a weight fraction of the

hydrophobic end groups $<10 \mathrm{wt} \%$ ) are directly dissolved in water. The homopolymers,

poly[2-(diethylamino)ethyl methacrylate], poly( $\mathrm{N}$-isopropylacrylamide), and 


\section{sumensus small}

poly(ethoxyethylacrylate) are synthesized by reversible addition-fragmentation chaintransfer

(RAFT) polymerization using $\mathrm{S}^{\prime} \quad$-1-dodecyl-( $\left.\mathrm{S}^{\prime} \quad\right)-\left(\alpha, \alpha^{\prime} \quad\right.$-dimethyl- $\alpha^{\prime} \quad$ ' -acetic acid) trithiocarbonate (DDMAT) and its derivatives as chain transfer agents (CTAs).

A range of polyelectrolyte homopolymers with different terminal groups are designed and synthesized, which under acidic aqueous solution direct the self-assembly to form well-defi ned nanostructures. This assembly behavior was also observed for neutral polymers, and it was determined that the structure of the hydrophobic end groups (and thus choice of RAFT CTA) are very important in facilitating this unusual selfassembly behavior of hydrophilic homopolymers. It is proposed that the functionality of commonly used CTAs such as DDMAT, can affect the solution association of the resultant homopolymers and can in fact afford $\mathrm{ABA}{ }^{\prime}$ type polymers, which can undergo self-assembly to form higher-order nanostructures.

\section{Introduction}

It has been well documented in recent decades that the self-assembly of amphiphilic block copolymers can form a range of different nanostructures such as spherical micelles, vesicles and cylinders. ${ }^{1-4}$ Even though self-assembly is influenced by a number of factors, ${ }^{5,6}$ a suitable hydrophilic-hydrophobic balance in the amphiphilic polymer is required to form higher order structures and the morphology afforded depends on this ratio which is classically described as the dimensionless 'packing parameter', $p .{ }^{7}$ Overall the factors which control the morphology of the resultant nanostructure include the chemical structure of the copolymer, the hydrophobic fraction, copolymer concentration in solution and the solvent properties. The hydrophobic fraction in the polymer is proposed to be perhaps the important parameter in the self-assembly process. As a general rule, copolymers with $<50$ vol. $\%$ hydrophobic block usually form micelles; copolymers with $50 \sim 66$ vol.\% hydrophobic block usually favor 


\section{scrims small}

vesicle formation; those with more than 25 vol. \% of hydrophobic proportion may form vesicles, inverted micro structures, other complex structures, and finally macroscopic precipitates. That is to say, polymers with a low hydrophobic content favor spherical micelle structures in polar solvents where the hydrophobic portion stays inside as a core and the hydrophilic corona is expressed from the core to stabilize the colloidal structure. An increase in the relative hydrophobicity usually leads to the formation of nanostructure morphologies such as polymer vesicles which have a hydrophobic membrane and the hydrophilic coronas are expressed inwards and outwards to form a hollow bilayer type structure. The preparation of vesicles is an area of great interest as they are proposed to be suitable as synthetic models of living cells or for the simultaneous encapsulation of hydrophobic molecules such as drugs in their membrane and hydrophilic species in the inner water pool cavity.

Recently, a number of reports have utilized homopolymers for the formation of vesicles using conventional self-assembly approaches. For example, Cha et al. reported that positively charged poly(L-lysine) polyelectrolytes could interact with negatively charged semiconductor nanocrystals to cooperatively self-assembly into vesicles in water with quantum dots in the vesicle membrane. ${ }^{8}$ It should be noted that in these cases, the poly(L-lysine) homopolymer itself does not form vesicles in water. In addition, Thayumanavan et al. have reported that one amphiphilic polyacrylamide homopolymer (Mn ca. $10 \mathrm{kDa}$ ) whose repeating unit consisted of a grafted relatively long hydrophobic chain ( 8 carbon atoms) and a short hydrophilic head group (one carboxylic acid group) can form vesicles in water whereas homopolymers with a shorter or longer hydrophobic chain in the repeating unit form spherical micelles. ${ }^{9}$ Additionally, hydrophilically modified comb-like polybutadienes with a hydrophilic fraction of 0.11-0.55 have also been explored for the synthesis of vesicle-type structures. All these previous examples of homopolymers self-assembly have a hydrophobicity fraction which predicts vesicle formation, regardless of whether the hydrophobicity originates from the 


\section{sumesustomall}

polymer backbone or side chains. However in recent years there has been increasing interest in using competing mechanisms for the self assembly of well-defined aggregates such as cylinders and vesicles. These include the exploration of using crystallization driven self assembly, ${ }^{10}$ electrostatic interactions, ${ }^{11}$ architecturally complex polymers ${ }^{12}$ or mid block interactions $\{$ Kim, $2009 \# 20\}$ for the well-defined assembly of polymers into morphologies not predicted based on packing parameter considerations.

In this work, we were interested in exploring the effect of end groups on the self-assembly of hydrophilic block copolymers. It is well-known that the introduction of hydrophobic end groups in thermally responsive polymers such as NIPAM has an effect on the LCST behavior ${ }^{13}$ but the effect of polymer end group on self assembly has been less well-established. In particular given the recent advances in controlled radical polymerization (CRP) techniques the ability to tune and tailor the end group has now been fully realized. This is especially true for polymers prepared by reversible addition-fragmentation chain transfer polymerization $(\text { RAFT })^{14-16}$ in which both $\alpha$ and $\omega$ chain end functionality can be incorporated into the chain transfer agent or post-polymerization using established end group modification methods. ${ }^{17,18}$ The effect of RAFT end group on the self-assembly has seldom been considered in the past due to the very small weight fraction compared to the polymer chain. A very recent paper by Davies and Lowe, highlights the influence of end group interactions on the self assembly of hydrophilic polymers. ${ }^{19}$ Given that a commonly utilized chain transfer agent is $S^{\prime}$-1-dodecyl$\left(S^{\prime}\right)$-( $\alpha, \alpha^{\prime}$-dimethyl- $\alpha^{\prime \prime}$-acetic acid) trithiocarbonate (DDMAT) which contains a dodecyl chain as part of the $\mathrm{Z}$ group we propose the choice of RAFT group will affect the self assembly of the resultant polymer.

In this work we report well-defined vesicle formation from the self-assembly of a highly hydrophilic and charged homopolymer in acidic water with hydrophobic fractions (which originate from the end group functionality) as low as $2 \mathrm{wt} \%$. Our findings lead us to propose 


\section{sumensusmall}

that vesicle formation from highly hydrophilic and charged homopolymers does not obey the traditional rules of polymer self-assembly and instead a focus on the subtle effects of polymer end groups should be considered rather than just considerations of the hydrophilic fraction. These results are important as many biological molecules such as polypeptides, proteins and DNA are polyelectrolytes and are charged when dissociated in water and hence understanding the driving forces and influences on self assembly are very important in these systems. Furthermore, charged molecular chains, commonly present in soft matter systems, play a fundamental role in determining structure, stability and the interactions of various molecular assemblies. A new understanding of their assembly processes is proposed to be of particular interest to scientists in many fields such as polymer science, colloidal and material science and even bioengineering.

\section{Results and Discussion}

\subsection{Design and Synthesis of Highly Hydrophilic Homopolymers}

Following on from some initial work in the area of diblock copolymer self-assembly we found that the nature of the hydrophobic end groups in these polymers are key in determining the resultant self-assembly behavior. ${ }^{20}$ To explore this effect in polyelectrolyte homopolymers a range of PDEA homopolymers with different end groups were designed and synthesized by RAFT, as shown in Scheme 1. All these homopolymers were protonated by $\mathrm{HCl}$ aqueous solution during the purification process to afford homopolymer polyelectrolytes.

\section{Scheme 1.}

Polymers 1-3 in Figure 1 were synthesized by the RAFT polymerization of DEA with carefully chosen $\alpha$ and $\omega$ terminal hydrophobic groups introduced by choice of chain transfer 


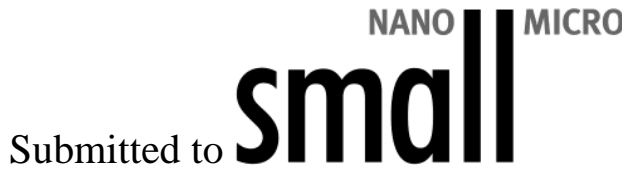

agent which were all based around the commonly used DDMAT structure. Specifically the effect of the long dodecyl chain which is often employed as a $\mathrm{Z}$ group in trithiocarbonates was explored as the effect of different hydrophobic R groups. Polymers 4 and $\mathbf{5}$ were synthesized by atom transfer radical polymerization (ATRP) with only one terminal hydrophobic end group. Polymer $\mathbf{1}$ has a hydrophobic, fluorescent pyrene group (Py) at the $\alpha$ position and a hydrophobic dodecyl (D) group at $\omega$ position. In polymer 2 the pyrene group was replaced by $-\mathrm{C}\left(\mathrm{CH}_{3}\right)_{2} \mathrm{COOH}(\mathrm{C})$, which was hydrophilic above neutral $\mathrm{pH}$ whereas hydrophobic at low $\mathrm{pH}$. Then in polymer $\mathbf{3}$ the $\mathrm{C}$ group was replaced by an ethyl ester functionality $-\mathrm{C}\left(\mathrm{CH}_{3}\right)_{2} \mathrm{COO}-\mathrm{CH}_{2} \mathrm{CH}_{3}$ (E) which is permanently hydrophobic and not $\mathrm{pH}$ sensitive. Polymers $\mathbf{4}$ and $\mathbf{5}$ only have a single hydrophobic group either Py or E, respectively as their chain end and were used as control systems. Table S1 in the supporting information reports the molecular weights and molecular weight distributions of the deprotonated PDEA homopolymers (1-5). A non-charged polymer, poly(N-isopropylacrylamide) (PNIPAM) was also synthesized with a Py and a D group (6) or with a C and D group (7) as additional controls. Given the difficulty in determining the molecular weight by GPC analysis the molecular weight of the polymers were calculated by comparing the integrated areas of pyrene end groups (peak $\mathrm{j}$ ) and PDEA side chains such as peaks $\mathrm{b}$ and $\mathrm{c}$ in a good solvent for all segments of the polymer chain (see Figure 2). The molecular weight and molecular weight distribution, as well as the weight fraction of the end group in the whole polymer are summarized in the Supporting Information (SI) Table S1. The weight percentage of the end groups in the homopolymer are all very low, ranging between 1-4\% except for polymer $\mathbf{1 . 1}$ which has a higher weight percentage of $10.2 \%$.

\section{Figure 1.}




\section{smallio}

\subsection{Self-Assembly of Homopolymers.}

The self-assembly of the homopolymer was easily performed by dissolving the homopolymer in acidic water at $\mathrm{pH} 2$ at ca. $5 \mathrm{mg} / \mathrm{mL}$. The self assembly of the polymers $\mathbf{1 - 5}$ was initially studied by analysis of the ${ }^{1} \mathrm{H}$ NMR spectra. In the case of homopolymers with hydrophobic end groups at both the $\alpha$ and $\omega$ positions (1-3) a strong attenuation of their end groups upon dissolution in acidic water was observed. A typical ${ }^{1} \mathrm{H}$ NMR spectrum of Py-PDEA $\mathrm{A}_{76}$-D homopolymer (1.2) in $\mathrm{D}_{2} \mathrm{O}$ at $\mathrm{pH} 3$ is shown in Figure 2. The strong attenuation of Py and $\mathrm{D}$ groups suggests the hydrophobic terminal groups are aggregated together in the polar hydrophilic solution. In contrast, homopolymers (4 and 5) with only one hydrophobic end group do not behave in this manner and instead no significant attenuation of pyrene signal was found from the ${ }^{1} \mathrm{H}$ NMR spectrum of Py-PDEA ${ }_{100}-\mathrm{Br}(4)$ in $\mathrm{D}_{2} \mathrm{O}$ at $\mathrm{pH} 3$ compared with the spectrum in its good solvent, $\mathrm{CDCl}_{3} / \mathrm{MeOD}$, as shown in Figure 2 . The close proximity of the two hydrophobic end groups in aqueous solution was confirmed through identification of a cross peak in the 2D-NOESY NMR spectra (Figure S1). This suggests that for the homopolymers with dual hydrophobic groups self assembly into higher order structures may be occurring in water whereas the homopolymers with a signal hydrophobic end group show no evidence of assembly under these conditions.

\section{Figure 2.}

The solution properties of the homopolymers in acidic water were compared and digital photos of the different homopolymer solutions are shown in Figure 3. Comparing the three vesicle solutions made from Py-PDEA $-\mathrm{D}(\mathbf{1 . 1}, 1.2$ and 1.4) with the same pyrene and dodecyl end groups ( $x=23,76$ and 150), the turbidity of the vesicle solution was observed to decrease with the increase of PDEA chain length, which suggests a decrease in particle size. 
Comparing the vesicle solutions of Py-PDEA $76^{-D}$ (1.2) and C-PDEA76-D (2), the vesicle solution with pyrene end group is more turbid than that with isobutyric acid groups, as a result of the higher hydrophobicity (rather than hydrophobic fraction) of pyrene compared to isobutyric acid group. The solution color of E-PDEA $74-\mathrm{D}$ (3) (not shown) is a slightly more turbid than that of $\mathbf{2}$ but less turbid then $\mathbf{1 . 2}$ indicating that they are immediate in size at the same concentration. This is consistent with the proposed order of hydrophobicity of the end group: $\mathrm{C}<\mathrm{E}<\mathrm{Py}$, in which the most hydrophobic end group affords the largest particles. As a control, homopolymers 4 and 5 which both only have a single hydrophobic end group were compared to the above results and it is clear that these solutions are similar to that of pure water, indicating that no aggregates were detected. This further indicates that dual hydrophobic end groups are required to affect the self-assembly of this charged hydrophilic homopolymer.

\section{Figure 3.}

\subsection{Dynamic Light Scattering (DLS) Studies of Self-Assemblies}

DLS studies confirmed the above empirical observations and indicated the formation of regular structures upon the self-assembly of the homopolymers with two hydrophobic end groups (1-3) whereas no regular self-assemblies were found for homopolymers containing only one hydrophobic end group (4 and 5) (Table 1). DLS is a technique used to determine particle size by examining the diffusion rates (i.e. Brownian motion) of suspended particles. The rate of signal change depends on the rate of change of the particles position, with large (hence slow moving) particles leading to slow fluctuations and small (hence fast moving) particles leading to fast fluctuations. As shown in Figure S1, the correlation functions revealed that when the concentration of polymer 1.2 is $0.1 \mathrm{mg} / \mathrm{mL}$, the measured correlation 


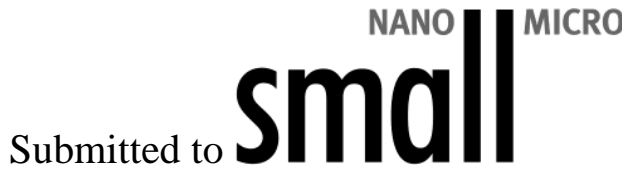

function is too poor for Cumulant analysis yet improves upon increasing the polymer concentration. The correlation curve at $0.1 \mathrm{mg} / \mathrm{mL}$ decays to baseline within $500 \mu \mathrm{s}$, indicating the smaller and hence faster diffusing free polymer chains or ill-defined loose aggregates. However, the larger and slower diffusing particles prepared at higher polymer concentration $(>1.0 \mathrm{mg} / \mathrm{mL})$ requires nearly $2000 \mu$ s before correlation in the signal is lost. The data quality from polymers 4 and 5 even at higher concentration $(20 \mathrm{mg} / \mathrm{mL})$ were still too poor to fit a Cumulant analysis, which means no self-assembly was observed for the homopolymers with only a single end group.

\section{Table 1.}

The correlation curve is a result of all the information regarding the diffusion of particles being measured. The diffusion coefficient $(D)$ is calculated by fitting the correlation curve to an exponential function, with D being proportional to the lifetime of the exponential decay (Figure $\mathrm{S} 2)$. The hydrodynamic radius $\left(\mathrm{R}_{\mathrm{h}}\right)$ is then calculated from the diffusion coefficient (D) using the Stokes-Einstein equation. According to this equation, DLS analysis revealed that the hydrodynamic diameters $\left(D_{h}\right)$ of the structures prepared at $5.0 \mathrm{mg} / \mathrm{mL}$ are 255,150 , 128 and $109 \mathrm{~nm}$, respectively when the degree of polymerization of DEA increased from 23, 76 to 122 to 150 (going from polymers 1.1 to $\mathbf{1 . 4}$ ) (Table 1). The above results are perhaps related to the decrease in the relative ratio of hydrophobic end groups to hydrophilic polyelectrolyte upon increasing the polymer chain length. For comparison the weight percentage of the hydrophobic end groups in $\mathbf{1 . 1}$ is $\mathbf{1 0 . 2}$, with this value decreasing to 3.32 wt $\%$ in $\mathbf{1 . 2}$, to 2.09 in $\mathbf{1 . 3}$ and $1.71 \mathrm{wt} \%$ in $\mathbf{1 . 4}$. In addition, the nature of the hydrophobic end group was found to affect the final morphology and particle size cf. the difference in size observed for $\mathbf{1 . 2}$ versus $\mathbf{2}$ and $\mathbf{3}$ which have very similar nanostructures sizes. The distribution 


\section{smallio}

of nanostructures for $\mathbf{2}$ is surprisingly high compared to the other assembly forming systems, which will be discussed later.

\subsection{TEM Analysis}

To further examine the structure of the assemblies formed from polymers 1-3, traditional TEM without or with a range of stains and techniques, as well as cryo-TEM were used to image the particles formed from these homopolymers. Initially a vesicular morphology was suggested by traditional TEM analysis of self-assemblies from polymer 1.2, 2 and $\mathbf{3}$ at 5.0 $\mathrm{mg} / \mathrm{ml}$ (Figure 4). The sample was viewed by traditional TEM directly on the discharged carbon film of TEM grid without any staining. It is clear that the dark circles represent the vesicle membrane. Furthermore, cryo-TEM analysis was used to confirm the vesicle structure. Typical cryo-TEM images of homopolymer $\mathbf{1 . 2}$ (at $5.0 \mathrm{mg} / \mathrm{mL}$ ) vesicles are shown in Figure WHAT. The central darker area clearly shows two vesicle membranes adhered together during cryo-TEM analysis.

\section{Figure 4.}

\subsection{Effect of Homopolymer Concentration}

The PDEA homopolymers were dissolved in water at $\mathrm{pH} 2$ at a range of concentrations from 0.1 to $10 \mathrm{mg} / \mathrm{mL}$. As shown in Figure 5, for homopolymer 1.2 (Py-PDEA 76 -D), DLS studies revealed that large nanostructures $>100 \mathrm{~nm}$ formed when the polymer concentration was higher than $1.0 \mathrm{mg} / \mathrm{mL}$, which alongside the TEM analysis was attributed to the formation of vesicles. The correlation functions of polymer vesicles are well fitted by Cumulants method and the size averaged by intensity, volume and number are reasonably consistent (see Figure S2 and Table S2). Polymer vesicles prepared at $10.0,5.0$ and $1.0 \mathrm{mg} / \mathrm{mL}$ were well-defined 


\section{smallio}

and the vesicle size decreases steadily with a decrease of polymer concentration from 189 to $94 \mathrm{~nm}$. When the concentration was $0.5 \mathrm{mg} / \mathrm{mL}$, the majority of the assemblies are proposed to be polymer micelles of ca. $30 \mathrm{~nm}$ (Figure 5), suggesting the transition polymer concentration from vesicles to micelles upon dilution of the sample. However, in the intensity-averaged plot (see Figure S3), the there appears to be a mixture of structures, indicating a larger structures are also present in the sample.

\section{Figure 5.}

The micelle solution at $0.5 \mathrm{mg} / \mathrm{mL}$ of $\mathbf{1 . 2}$ was also examined by cryo-TEM analysis. As shown in Figure 6, the spherical shape is clear and the overall diameter is $\sim 35 \mathrm{~nm}$, which is consistent with the DLS results ( $\sim 31 \mathrm{~nm})$. Further decreasing in the polymer concentration to $0.1 \mathrm{mg} / \mathrm{mL}$ affords ill-defined assemblies by DLS (intensity averaged) and indicates that this may be below or close to the critical micelle concentration $(\mathrm{cmc})$ of the homopolymers. This was confirmed by determining the cmc of the homopolymers using PNA as a probe (Figure

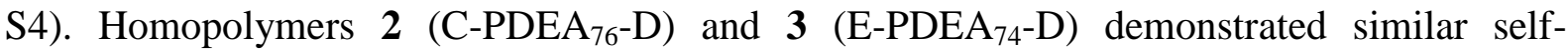
assembly behavior to polymer $\mathbf{1 . 2}$ upon changing the polymer concentration, with a decrease in nanostructure size with decreasing concentration (Figures S5 and S6). A summary of the DLS studies of polymers 1.2, 2 and $\mathbf{3}$ are shown in Figure 7 (see also Table S2). These observations can be related to the cmc of the polymers and indicates that for the polymer with the most hydrophobic end $\alpha$-group (1.2) has the lowest cmc and compared to polymers 2 and 3. Indeed polymer 2 which contains the hydrophobic carboxylic acid end group displays a rather high $\mathrm{cmc}$ in the region of $1 \mathrm{mg} / \mathrm{mL}$ compared to the other polymers studied in this work. 


\section{statillio}

\section{Figure 6.}

Figure 7.

\subsection{Static Light Scattering (SLS) Characterization}

The radius of gyration, $R_{\mathrm{g}}$, is defined as the mass weighted average distance from the centre of mass to each mass element, which was measured by monitoring the angular dependence of the sample scattering intensity in SLS. Whereas the hydrodynamic radius, $R_{\mathrm{h}}$, is the representative of the size of a hard sphere that diffuses at the same rate as the particle being measured, which is measured by DLS. It is well known that the structure of the selfassemblies can be roughly estimated by $R_{\mathrm{g}} / R_{\mathrm{h}}$ ratio. For hard spheres, the theoretical value is 0.77 ; for vesicles, the value is 1.0 ; for cylinders, the value is $c a$. 2 . Hence to confirm a vesicular morphology for the nanostructures formed from polymer 1.2 we performed SLS measurements at a range of concentrations and angles. Given the concentration size dependence observed for these samples often a narrow window of concentrations was required to ensure reproducible and meaningful results. The data was analysed using a Zimm plot and the resultant $R_{\mathrm{g}} / R_{\mathrm{h}}$ value was calculated to be 0.99 , which is very close to the theoretical value of 1.0 for vesicles. This was repeated for polymers $\mathbf{2}$ and $\mathbf{3}$, and the calculated the $R_{\mathrm{g}} / R_{\mathrm{h}}$ values were 1.16 and 1.12 respectively which are close to 1.0 and thus suggested a vesicular morphology (Figure S7). We proposed based on the DLS, SLS, TEM and NMR data that the homopolymer vesicle has a positively charged folded coronas with hydrophobic end groups forming the vesicle membrane.

\subsection{Effect of The Polymer Type}




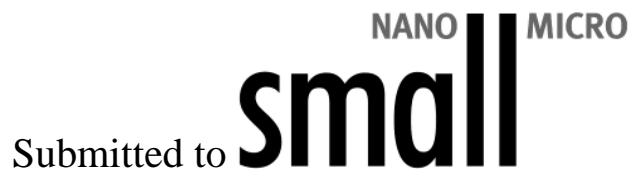

The effect of the nature of the polymer chain on the self-assembly was also studied. Thermoresponsive poly( $N$-isopropylacrylamide) (PNIPAM) homopolymers with different end groups, Py-PNIPAM ${ }_{72}-\mathrm{D}(6)$ and C-PNIPAM $115-\mathrm{D}$ (7) were synthesized by RAFT and their self-assemblies at $25{ }^{\circ} \mathrm{C}$ (Table S1) and different concentrations were investigated by DLS analysis. At $25{ }^{\circ} \mathrm{C}$ the PNIPAM homopolymer is highly hydrophilic and can be dissolved in water directly (Figure S9). Upon dissolution the Z-averaged diameter of particles from polymer 6 at $\sim$ pH 6 was $\sim 200 \mathrm{~nm}$ by DLS at $5.0 \mathrm{mg} / \mathrm{ml}$ with a PDI of 0.101 (Figure S10). The particles were furthered characterized by TEM and a vesicle structure was confirmed (Figure 8). Polymer 7 was also dissolved in water at $\mathrm{pH} 6$ at $5.0 \mathrm{mg} / \mathrm{mL}$ however no ordered self-assembly was found at this $\mathrm{pH}$ due to the singly hydrophobic end group at this $\mathrm{pH}$. However upon decreasing the solution $\mathrm{pH}$ to 2 relatively small particles (41 nm, PDI 0.349 ) were observed, which were considered to be polymer micelles (Figure S11). This confirms that the hydrophobic dual end groups are very important for the vesicle and micelle formation. With Py and D end groups, both PDEA and PNIPAM homopolymers can self-assemble into vesicles. However, with $\mathrm{C}$ and D end groups, PDEA homopolymers form vesicles whereas PNIPAM homopolymers only form micelles, rather than vesicles. This may in part be due to the polyelectrolyte nature of the PDEA which prevents the formation of curvature structures in favor of bilayers. This means that the polymer chains itself may affect the self-assembly besides the end groups.

\section{Figure 8.}

\section{Conclusions}

In summary, a new class of polyamine homopolymers was synthesized by RAFT and ATRP. Those highly hydrophilic homopolymers (upon protonation) with two small hydrophobic end 


\section{sumests small}

groups in both head and tail positions can be directly dissolved in acidic water to form polymer vesicles, which was confirmed by ${ }^{1} \mathrm{H}$ NMR, DLS, SLS and TEM studies. The homopolymers only with single small hydrophobic end group couldn't self-assemble into regular nanostructure in aqueous solution. The vesicles size decreases with the decrease of the polymer concentration or the hydrophobicity of the end groups. Due to their homopolymer polyelectrolytes nature, they may be applied to the mimic of positively charged DNA selfassembly or used as the gene delivery vehicle or drug container. In addition, highly hydrophilic PNIPAM with two small hydrophobic end groups is confirmed to form vesicles at similar conditions. Compared to traditional self-assembly from block copolymers, the homopolymer is simpler and has many advantages in the synthesis, characterization and application. The versatile available CTAs and monomers make the future of homopolymer synthesis and self-assembly to nanostructures very promising.

\section{Experimental Section}

Materials. AIBN (2,2'-Azobis(2-methylpropionitrile)) was recrystallized from a 9:1 mixture of hexanes/acetone and stored in the dark at $4{ }^{\circ} \mathrm{C}$. Diethyl aminomethylate (DEA) was purified via vacuum distillation over $\mathrm{CaH}_{2}$ and then stored at $4{ }^{\circ} \mathrm{C}$. $N$-iso-propyl acrylamide (NIPAM) was recrystallized from a 9:1 mixture of hexanes/acetone and stored at $4{ }^{\circ} \mathrm{C}$. Other reagents were purchased from Aldrich and used as received. DDMAT ( $S^{\prime}$-1-dodecyl- $\left(S^{\prime}\right)$ $(\alpha, \alpha$-dimethyl- $\alpha$ "-acetic acid) trithiocarbonate) and ethyl-DDMAT and pyrene-DDMAT were synthesized as reported previously. ${ }^{20,}{ }^{21}$ When used, dry solvents were collected and used directly from an in house drying and degassing solvent tower delivery system. Dialysis tubing was purchased from Medicell International Ltd. with a molecular weight cut-off of $1,000 \mathrm{Da}$. 


\section{smallio}

Characterization. THF GPC. The molecular weight distributions of the PDEA homopolymers were assessed at $30{ }^{\circ} \mathrm{C}$ using a Polymer Laboratories PL-GPC50 Integrated GPC system equipped with a Polymer Laboratories pump, a PLgel $5 \mu \mathrm{m}$ MIXED-C column $(300 \times 7.5$ mm), a WellChrom K-2301 refractive index detector, a viscometry detector and a PD 2020 light scattering detector. The calibration was carried out using six poly(methyl methacrylate) standards with Mp values ranging from 1,310 to 211,400 Da. The eluent was THF containing $2.0 \%(\mathrm{v} / \mathrm{v})$ TEA and $0.05 \%(\mathrm{w} / \mathrm{v})$ BHT and the flow rate was $1.0 \mathrm{~mL} / \mathrm{min}$. The data were processed using Cirrus GPC offline GPC/SEC software (version 2.0).

${ }^{1} H$ NMR spectra. We recorded the spectra using a Bruker AV $400(400 \mathrm{MHz})$ spectrometer at ambient temperature using either $\mathrm{CDCl}_{3}, \mathrm{CD}_{3} \mathrm{OD} / \mathrm{CDCl}_{3}, \mathrm{D}_{2} \mathrm{O} / \mathrm{H}_{2} \mathrm{O}$ or $\mathrm{D}_{2} \mathrm{O}$ as solvents. When $\mathrm{D}_{2} \mathrm{O} / \mathrm{H}_{2} \mathrm{O}$ was used as solvent, the ${ }^{1} \mathrm{H}$ NMR experiment with water suppression' option was selected.

TEM. TEM images were obtained using a JEOL electron microscope operating at $200 \mathrm{kV}$ equipped with a LaB6 gun and a Gatan digital camera. To prepare TEM samples, $5 \mu \mathrm{L}$ of an aqueous vesicle solution was placed on a carbon-coated copper grid for $60 \mathrm{~s}$, and the water droplet was removed by a vacuum absorption.

Cryo TEM were performed by Dr Steve Furzeland and Derek Atkins, Unilever. Sample preparation was carried out using a CryoPlunge 3 unit (Gatan Instruments) employing a double blot technique. $3 \mu 1$ of sample was pipetted onto a plasma etched (15secs) 400 mesh holey carbon grid (Agar Scientific) held in the plunge chamber at approx 90\% humidity. The samples were blotted, from both sides for $0.5,0.8$ or 1.0 second dependant on sample viscosity. The samples were then plunged into liquid ethane at a temperature of $-170{ }^{\circ} \mathrm{C}$. The 


\section{sonesestosmall}

grids were blotted to remove excess ethane then transferred, under liquid nitrogen to the cryo TEM specimen holder (Gatan 626 cryo holder) at $-170{ }^{\circ} \mathrm{C}$. Samples were examined using a Jeol 2100 TEM operated at 200KV and imaged using a Gatan Ultrascan 4000 camera and images captured using DigitalMicrograph software (Gatan).

$D L S$. DLS studies of aqueous vesicles were conducted over a range of solution $\mathrm{pH}$ at a fixed scattering angle of $173^{\circ}$. The data were processed by cumulants analysis of the experimental correlation function and vesicle diameters were calculated from the computed diffusion coefficients using the Stokes-Einstein equation. Each reported measurement was the average of three runs.

Static Light Scattering (SLS) Measurements. SLS analysis was performed on a Malvern Instruments Autosizer 4800 equipped with an APD detector and a Malvern 713250 ns 16-bit digital auto-correlator, using a $50 \mathrm{~mW}$ green incident laser beam. SLS data was collected for 4 or more different concentrations of the vesicles, 20 different angles for each concentration and was analyzed using the Zimm plot method on Malvern PSW0078 Advanced software to determine $\mathrm{Rg}$.

Synthesis of pyrene-based ATRP initiator. A flask with a magnetic flea was charged with 1pyrenemethanol (1.000 g; $4.219 \mathrm{mmol})$, anhydrous THF (30 mL), TEA (0.4744 g; 4.641 mmol). This flask was placed in the ice bath. 2-bromoisobutyryl bromide (1.089 g; 4.641 mmol) was then added dropwise. Precipitation was immediately observed. Five hours later, 1 $\mathrm{mL}$ of methanol was added and reacted for 20 minutes. THF and residual methanol was removed by rotator evaporator. The yellow crude product is solid. The black and viscous fluid is byproduct. Wash with $100 \mathrm{~mL}$ of $0.01 \mathrm{M} \mathrm{NaOH}$ water for $1 \mathrm{~h}$. Then wash with neutral 


\section{smallio}

water for 3 times. Recrystallize in methanol twice. Yellow product. Yield: $37 \%$. ${ }^{1} \mathrm{H}$ NMR spectrum: 8.35-8.0 (m, pyrene, 9H); $5.93\left(\mathrm{~s}, \mathrm{Py}_{-} \mathrm{CH}_{2}-\mathrm{O}, 2 \mathrm{H}\right) ; 1.94$ [s, OOC-C $\left(\mathrm{CH}_{3}\right)_{2} \mathrm{Br}, 6 \mathrm{H}$ ].

RAFT Synthesis of PDEA homopolymer. In a typical RAFT protocol, a flask with a magnetic flea and a rubber septum was charged with AIBN radical initiator (3.0 mg; 3.3×10-2 mmol), pyrene-DDMAT CTA $(94.0 \mathrm{mg} ; 0.162 \mathrm{mmol})$, DEA monomer $(3.039 \mathrm{~g} ; 16.2 \mathrm{mmol})$ and dioxane $(8.12 \mathrm{~mL})$. This solution was deoxygenated using a $\mathrm{N}_{2}$ sparge for 30 minutes before heating at $80{ }^{\circ} \mathrm{C}$ under a nitrogen atmosphere. The [DEA]: [pyrere-DDMAT]: [AIBN] relative molar ratios were 100: 1: 0.2 . After $4.5 \mathrm{~h}$ the monomer conversion is $76 \%$ as judged by $1 \mathrm{H}$ NMR. The final reaction solution was diluted with acetone and $0.01 \mathrm{M} \mathrm{HCl}$ aqueous solution and then purified by dialysis against water at $\mathrm{pH} 2-4$. A fine white powder was obtained after freeze-drying. A ${ }^{1} \mathrm{H}$ NMR spectrum of the purified block copolymer was recorded using a $\mathrm{D}_{2} \mathrm{O}$ as solvent.

ATRP Synthesis of PDEA homopolymer. In a typical ATRP protocol, a flask with a magnetic flea and a rubber septum was charged with ethyl 2-bromoisobutyrate initiator $(50.0 \mathrm{mg} ; 0.260$ mmol), bpy ligand ( $80.1 \mathrm{mg} ; 0.52 \mathrm{mmol})$, DEA monomer (4.077 g; $21.8 \mathrm{mmol})$ and methanol $(10 \mathrm{~mL})$. This solution was deoxygenated using a $\mathrm{N}_{2}$ sparge for 30 minutes before adding $\mathrm{Cu}(\mathrm{I}) \mathrm{Br}(36.8 \mathrm{mg}, 0.26 \mathrm{mmol})$. The [DEA]: [2-bromoisobutyrate]: [CuBr]: [bpy] relative molar ratios were 85: 1: 1: 2 . The DEA polymerization was conducted under a nitrogen atmosphere at $20{ }^{\circ} \mathrm{C}$. After $2.5 \mathrm{~h}$ and $20 \mathrm{~h}$, the monomer conversions are $50 \%$ and $96.5 \%$, respectively, as judged by ${ }^{1} \mathrm{H}$ NMR. The final reaction solution was diluted with acetone and $0.01 \mathrm{M} \mathrm{HCl}$ aqueous solution and purified by dialysis against water at $\mathrm{pH} 2-4$. A fine white powder was obtained after freeze-drying. A ${ }^{1} \mathrm{H}$ NMR spectrum of the purified block copolymer was recorded using a $\mathrm{D}_{2} \mathrm{O}$ as solvent. 


\section{smallio}

RAFT Synthesis of PNIPAM homopolymers. In a typical RAFT protocol, a flask with a magnetic flea and a rubber septum was charged with AIBN radical initiator $\left(2.0 \mathrm{mg} ; 2.2 \times 10^{-}\right.$ ${ }^{2}$ mmol), pyrene-DDMAT CTA (42.0 mg; $\left.7.2 \times 10^{-2} \mathrm{mmol}\right)$, NIPAM monomer $(0.653 \mathrm{~g}$; $5.77 \mathrm{mmol}$ ) and dioxane/DMF (2.8 $\mathrm{mL}$ each). This solution was deoxygenated using a $\mathrm{N}_{2}$ sparge for 60 minutes before heating at $65{ }^{\circ} \mathrm{C}$ under a nitrogen atmosphere. The [NIPAM]: [pyrere-DDMAT]: [AIBN] relative molar ratios were 100: 1: 0.3. After $5.5 \mathrm{~h}$ the monomer conversion is $75 \%$ and after $24 \mathrm{~h}$ the conversion is $95 \%$ as judged by ${ }^{1} \mathrm{H}$ NMR. The precipitation by diethyl ether in the presence of dry ice only yields $10 \%$ of product. Thus water was added into the polymer solution in DMF and then dialyzed against water. The fine powder was obtained by freeze dry. Yield: $78 \% .{ }^{1} \mathrm{H}$ NMR spectrum of the purified homopolymer was recorded using a DMSO- $\mathrm{d}_{6}$ or $\mathrm{D}_{2} \mathrm{O}$ as solvent to investigate the selfassembly.

Preparation of Vesicles. Typically PDEA homopolymer $(100.0 \mathrm{mg}$, varying at different copolymer concentrations) was dissolved into $10.00 \mathrm{~g}$ pure water at $\mathrm{pH} 2$ at room temperature to form vesicles directly. PNIPAM homopolymers were dissolved in neutral water, stirred for 2 hours.

\section{Acknowledgements}

The Leverhulme Trust is thanked for a post-doctoral fellowship for J.D. The EPSRC and Royal Society are thanked for support for R.K.O.R. The GPC used in this research was obtained, through Birmingham Science City: Innovative Uses for Advanced Materials in the Modern World (West Midlands Centre for Advanced Materials Project 2), with support from Advantage West Midlands (AWM) and part funded by the European Regional Development 


\section{suments small}

Fund (ERDF). We thank the Electron Microscopy Facility, Department of Biological Sciences,

University of Warwick (Welcome Trust grant reference: 055663/Z/98/Z) for cryo-instrument use and technical support from Mr Ian Portman.

\section{References}

1. M. Antonietti and S. Förster, Advanced Materials, 2003, 15, 1323-1333.

2. J. Du and R. K. O'Reilly, Soft Matter, 2009, 5, 3544-3561.

3. R. K. O'Reilly, C. J. Hawker and K. L. Wooley, Chem. Soc. Rev., 2006, 35, 1068-1083.

4. E. S. Read and S. P. Armes, Chem. Commun., 2007, 3021-3035.

5. H. Cui, Z. Chen, S. Zhong, K. L. Wooley and D. J. Pochan, Science, 2007, 317, 647650.

6. R. C. Hayward and D. J. Pochan, Macromolecules, 2010, 43, 3577-3584.

7. Isrelachvili, J. Chem. Soc., Faraday Trans., 1976, 72, 1525.

8. J. N. Cha, H. Birkedal, L. E. Euliss, M. H. Bartl, M. S. Wong, T. J. Deming and G. D. Stucky, Journal of the American Chemical Society, 2003, 125, 8285-8289.

9. S. Arumugam, D. R. Vutukuri, S. Thayumanavan and V. Ramamurthy, Journal of the American Chemical Society, 2005, 127, 13200-13206.

10. T. Gadt, N. S. Ieong, G. Cambridge, M. A. Winnik and I. Manners, Nat. Mater., 2009, 8, 144-150.

11. M. Lazzari and M. A. Quitela, Macromol. Rapid Commun., 2009, 30, 1785-1791.

12. Z. Li, E. Kesselman, Y. Talmon, M. A. Hillmyer and T. P. Lodge, Science, 2004, 306, 98-101.

13. S. Furyk, Y. Zhang, D. Ortiz-Acosta, P. S. Cremer and D. E. Bergbreiter, J. Polym. Sci. Part A: Polym. Chem., 2006, 44, 1492-1501.

14. J. Chiefari, Y. K. Chong, F. Ercole, J. Krstina, J. Jeffery, T. P. T. Le, R. T. A. Mayadunne, G. F. Meijs, C. L. Moad, G. Moad, E. Rizzardo and S. H. Thang, Macromolecules, 1998, 31, 5559-5562.

15. G. Moad, E. Rizzardo and S. H. Thang, Aust. J. Chem., 2005, 58, 379-410.

16. S. Perrier and P. Takolpuckdee, J. Polym. Sci. Part A: Polym. Chem., 2005, 43, 53475393.

17. G. Moad, Y. K. Chong, A. Postma, E. Rizzardo and S. H. Thang, Polymer, 2005, 46, 8458-8468.

18. H. Willcock and R. K. O'Reilly, Polym. Chem., 2010, 1, 149.

19. J. Xu, L. Tao, C. Boyer, A. B. Lowe and T. P. Davies, Macromolecules, 2011, DOI: 10.021/ma102386j.

20. J. Du and R. K. O'Reilly, Macromol. Chem. Phys., 2010, 211, 1530-1537.

21. J. Skey and R. K. O'Reilly, Chem. Commun., 2008, 4183-4185.

Received: ((will be filled in by the editorial staff))

Revised: ((will be filled in by the editorial staff))

Published online on ((will be filled in by the editorial staff)) 


\section{mater smans small}

Scheme 1.

$\mathrm{R}_{1}$-PDEA-R $2 \quad \mathrm{R}_{1}$-PNIPAM- $\mathrm{R}_{2}$
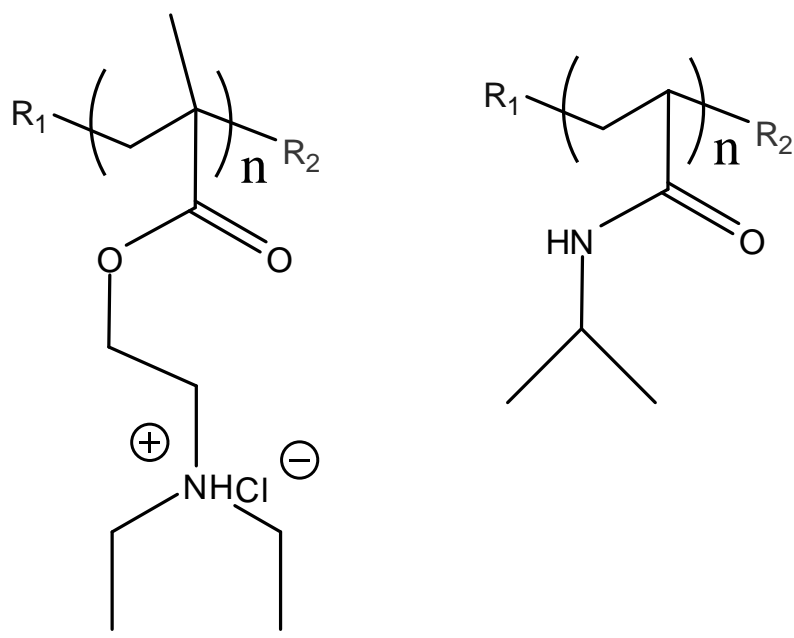

\begin{tabular}{|c|c|c|c|c|}
\hline & Polymer & $\mathrm{R}_{1}$ & $\mathrm{R}_{2}$ & $\mathrm{n}$ \\
\hline 1.1 & & & & 23 \\
\hline 1.2 & & & & 76 \\
\hline 1.3 & & & & 122 \\
\hline 1.4 & & & & 150 \\
\hline 2 & C-PDEA-D & & & 76 \\
\hline 3 & E-PDEA-D & & & 74 \\
\hline 4 & Py-PDEA-Br & & $\mathrm{Br}$ & 100 \\
\hline 5 & E-PDEA-Br & & $\mathrm{Br}$ & 82 \\
\hline
\end{tabular}




\section{suraments small}

6 Py-PNIPAM-D

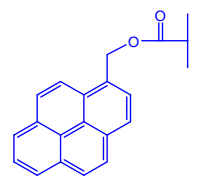

HO<smiles>CC(C)(C)SC(=S)S</smiles><smiles>CC(C)(SC(=S)S)C1CC1</smiles>

72

Figure 1. Homopolymers PDEA (1-5) and PNIPAM (6 and 7) with different end groups.

Dual hydrophobic end groups: vesicles
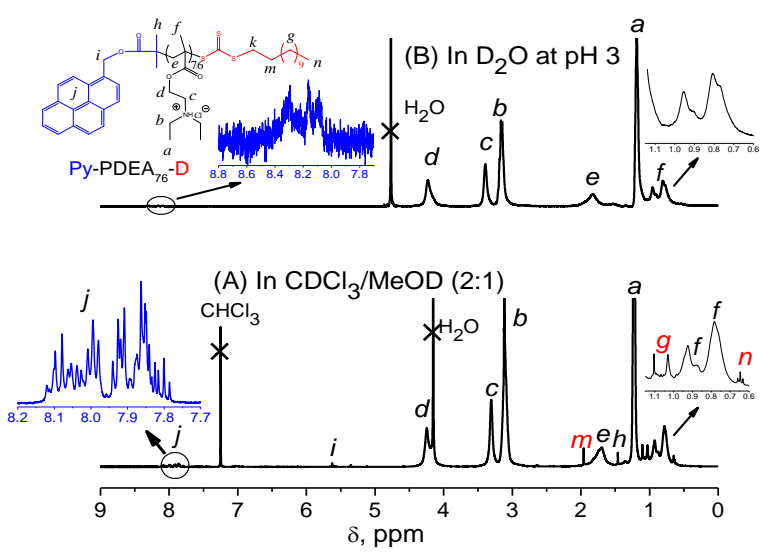

Single end group: no self-assembly

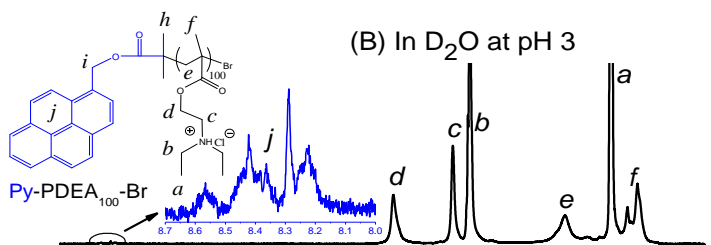

(A) In $\mathrm{CDCl}_{3} / \mathrm{MeOD}(2: 1)$

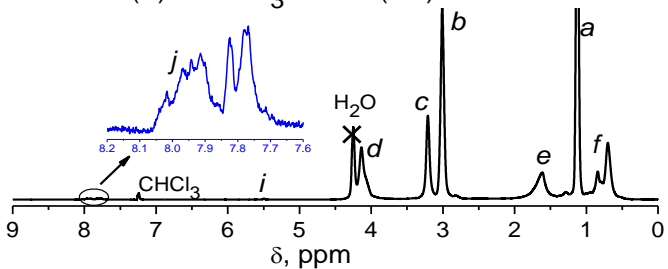

Figure 2. 1H NMR spectra of homopolymers with dual hydrophobic end groups versus a single hydrophobic end group at $5 \mathrm{mg} / \mathrm{mL}$ : Left: Homopolymer 1.2, Py-PDEA ${ }_{76}-\mathrm{D}$, with two hydrophobic end groups: (A) in $\mathrm{CDCl}_{3} / \mathrm{MeOD}(2: 1)$, a good solvent for both polymer and end groups; (B) in $\mathrm{D}_{2} \mathrm{O}$ at $\mathrm{pH} 3$, a non-solvent for the end groups. The signals from pyrene (peak $\mathrm{j}$, blue) and dodecyl (peaks $\mathrm{g}, \mathrm{n}$ and $\mathrm{m}$, red) in $\mathrm{CDCl}_{3} / \mathrm{MeOD}$ are visible but remarkably attenuated in $\mathrm{D} 2 \mathrm{O}$, indicating the aggregation of the pyrene and dodecyl groups in a polar hydrophobic environment. Right: Homopolymer 4, Py-PDEA ${ }_{100}-\mathrm{Br}$, with one hydrophobic end group: (A) in $\mathrm{CDCl}_{3} / \mathrm{MeOD}(2: 1)$; (B) in $\mathrm{D}_{2} \mathrm{O}$ at $\mathrm{pH}$ 3. The signals from pyrene (peak $\mathrm{j}$, blue) in $\mathrm{CDCl}_{3} / \mathrm{MeOD}$ are visible and no obvious attenuation in $\mathrm{D}_{2} \mathrm{O}$ was found, indicating loose or no aggregation of pyrene end group in a polar environment.

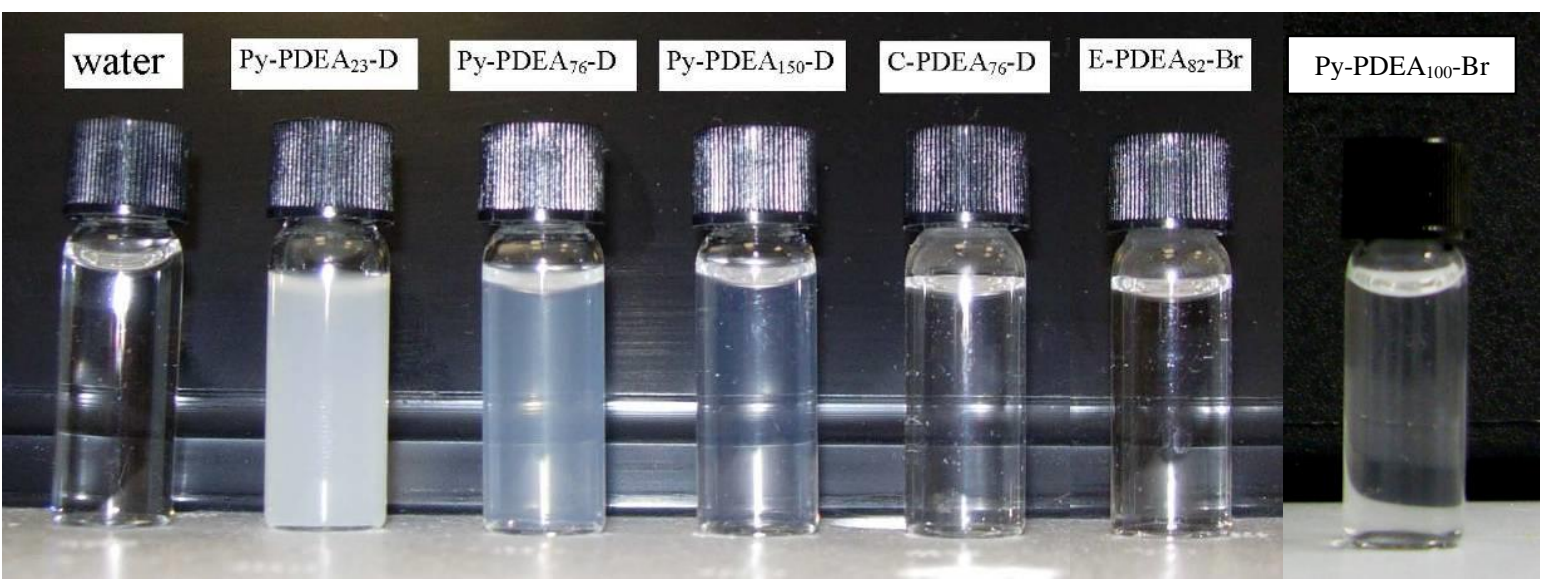


Figure 3. Digital camera image of vesicle solution by direct dissolution of different homopolymers in water at $\mathrm{pH} 2$ at $5.0 \mathrm{mg} / \mathrm{mL}$.

Table 1. DLS studies of vesicles by direct dissolution of PDEA homopolymers at $5.0 \mathrm{mg} / \mathrm{mL}$ at $\mathrm{pH} 2$ or PNIPAM homopolymer at $\mathrm{pH} 6$ in water.

\begin{tabular}{clll}
\hline Code & Polymer $^{a}$ & $\begin{array}{l}D_{\mathrm{h}} \\
(\mathrm{nm})^{b}\end{array}$ & PDI $^{c}$ \\
\hline $\mathbf{1 . 1}$ & Py-PDEA $_{23}$-D & 255 & 0.207 \\
$\mathbf{1 . 2}$ & Py-PDEA $_{76}$-D & 150 & 0.084 \\
$\mathbf{1 . 3}$ & Py-PDEA $_{122}-\mathrm{D}$ & 128 & 0.190 \\
$\mathbf{1 . 4}$ & Py-PDEA $_{150}-\mathrm{D}$ & 109 & 0.121 \\
$\mathbf{2}$ & C-PDEA $_{76^{-D}}$ & 107 & 0.307 \\
$\mathbf{3}$ & E-PDEA $_{74}-\mathrm{D}$ & 104 & 0.133 \\
$\mathbf{4}^{\boldsymbol{d}}$ & Py-PDEA $_{100}-\mathrm{Br}$ & - & - \\
$\mathbf{5}^{\boldsymbol{d}}$ & E-PDEA $_{82}-\mathrm{Br}$ & - & - \\
$\mathbf{6}$ & Py-PNIPAM $_{72}-\mathrm{D}$ & 202 & 0.101 \\
$\mathbf{7}$ & C-PNIPAM $_{115}-\mathrm{D}$ & 41 & 0.349 \\
\hline
\end{tabular}

[a] End groups: Py: pyrene; D: dodecyl carbonotrithiocarbonate; C: Isobutyric acid, which is protonated at low $\mathrm{pH}$; E: ethyl ester. [b] Z-averaged diameter by DLS by intensity. [c] Polydispersity of particles by DLS. [d] Data quality is too poor to be analyzed by Cumulant method.

Figure 4. Representative TEM images of 1.2, 2 and 3.

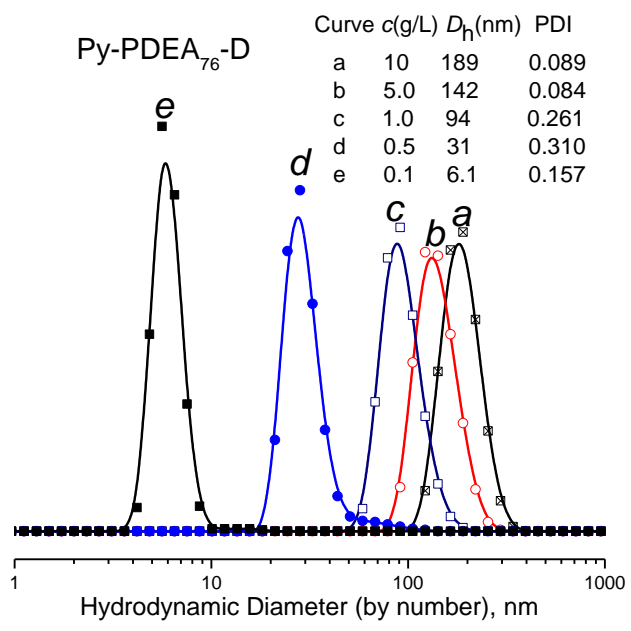

Figure 5. DLS studies of homopolymer vesicles prepared by direct dissolution of $\mathbf{1 . 2}$ (Py$\mathrm{PDEA}_{76}-\mathrm{D}$ ) in water at $\mathrm{pH} 2$ at different concentrations (the corresponding correlation functions and intensity-averaged size distribution are shown in SI Fig. S2 and Fig. S3, respectively). 


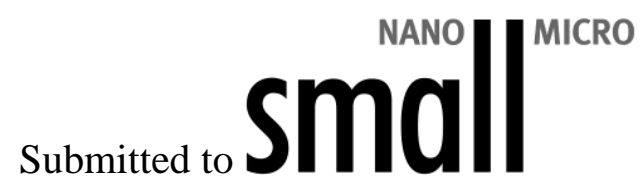

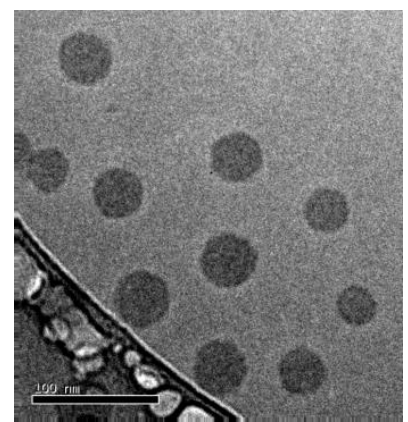

Figure 6. Representative cryo-TEM of polymer 1.2 assembled at $0.5 \mathrm{mg} / \mathrm{mL}$ and $\mathrm{pH}=2$.

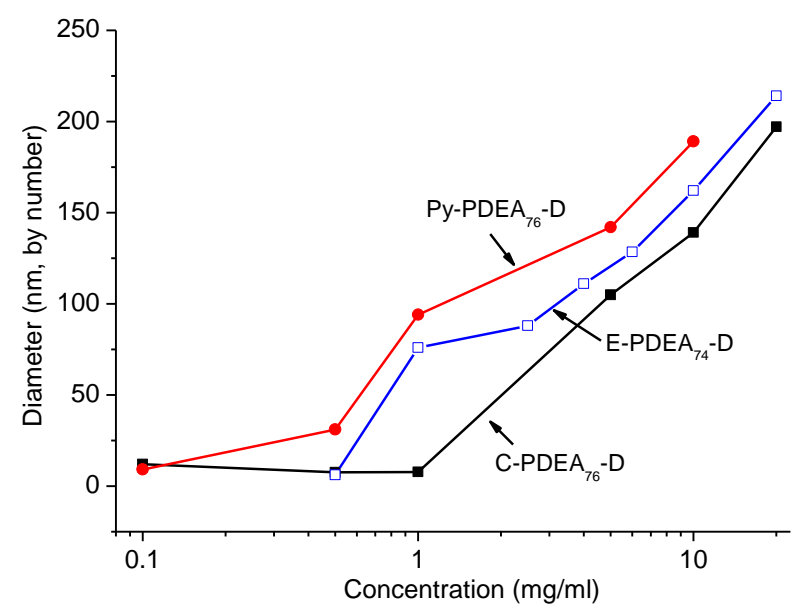

Figure 7. The particle diameter determined by DLS (by number) as a function of polymer concentration in water at $\mathrm{pH} 2$ for three PDEA homopolymers: 1.2, 2 and 3.
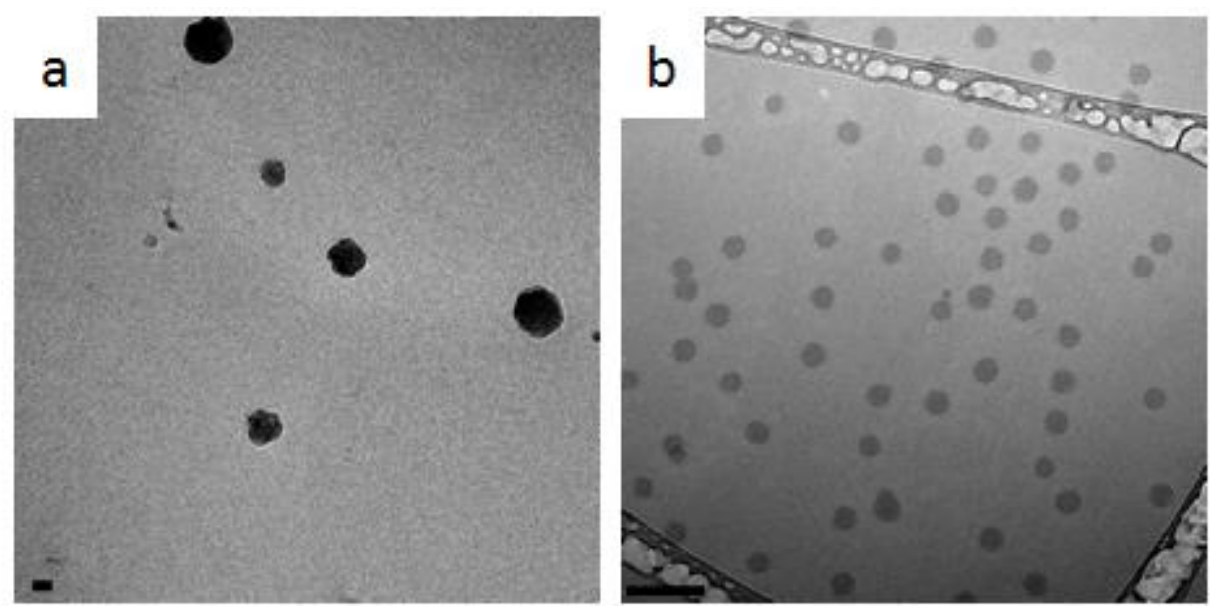

Figure 8. Representative cryo TEM images for a) 6 and b) 7 assembled at $5.0 \mathrm{mg} / \mathrm{mL}$. 
The table of contents entry should be fifty to sixty words long, written in the present tense, and refer to the chosen figure.

TOC Keyword

C. Author-Two, D. E. F. Author-Three, A. B. Corresponding Author*((same order as byline) $) \boldsymbol{\square}<\mathrm{B}>\ldots<\mathrm{B}>$

Title ((no stars))

ToC figure ((55 $\mathrm{mm}$ broad, $40 \mathrm{~mm}$ high $))$

Page Headings

Left page: $\quad$ First Author et al.

Right page: Title of manuscript (abbreviated) 


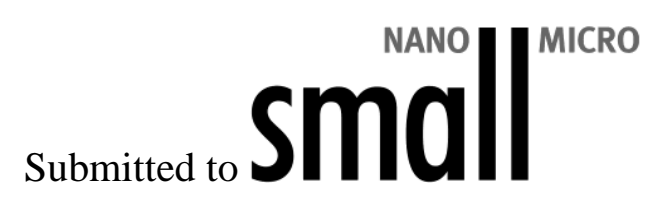

Supporting Information should be included here (for submission only; for publication, please provide Supporting Information as a separate PDF file). 